\title{
Profitability of Organic and Conventional Dairy Production with Different Dietary Proportions of High-Quality Grass Silage
}

\author{
M. Patel ${ }^{1}$, E. Wredle ${ }^{1}$, E. Spörndly ${ }^{1}$, J. Bertilsson ${ }^{1}$ and K.-I. Kumm². \\ ${ }^{1}$ Swedish University of Agricultural Sciences, Department of Animal Nutrition and \\ Management. Kungsängen Research Centre, SE-753 23 Uppsala, Sweden. \\ (mikaela.patel@slu.se) \\ ${ }^{2}$ Swedish University of Agricultural Sciences, Department of Animal Environment and \\ Health, P.O. Box 234, SE-532 23 Skara, Sweden.
}

\section{Implications}

Profitability in milk production was calculated using diets with three different proportions of high-quality grass silage. The treatments consisted of the same feeds, but differed in the dietary proportion of forage: low $(\mathrm{L})$, medium $(\mathrm{M})$ and high $(\mathrm{H})$, representing one conventional and two organic diets. The calculations were based on results in feed intake and milk production from a large dairy cow experiment. In the calculations, three different districts with different conditions for farming were used as models, and calculations were performed on two different herd sizes. In addition, current financial supports were both included and excluded in the calculations. The results showed only minor differences in profitability between using in average $60 \%$ or $70 \%$ of dry matter (DM) of silage in diets used in organic production. In conventional production, it was profitable to increase the average dietary proportion of silage from $50 \%$ to $60 \%$ of DM when the prices of concentrate and grains were as high as at the present. The results showed clear economic benefits of increasing the proportion of high-quality silage in conventional Scandinavian dairy cow diets up to levels similar to the standards of the organic production system.

\section{Background and objectives}

The feed costs constitute a large part of the dairy farmers' total expenses both in organic and conventional production systems, and it is important to find alternative feeds or diets that can be produced at lower costs. The price of grains is currently high, and has been fluctuating over the last few years, making it an erratic cost item for the farmer. Therefore, high-quality forage is likely to have a large economic potential in future milk production systems. The main objective of the present study was to relate the results from a dairy cow experiment with different proportions of grass silage in the diet (Patel 2012), to the profitability in dairy production. To make the economic assessments more applicable to on-farm situations, calculations were performed using three different districts with different conditions for farming: a plains district in the south of Sweden (SP), a forest dominated district in the southern part of Sweden (SF) and a forest dominated district in the north of Sweden (NF). The economic support from the European Union (EU) is high in the forest dominated districts due to fewer opportunities for agriculture and due to the interest in maintaining an open varied agricultural landscape. Calculations were also performed on two different herd sizes in order to investigate the impact of scale. Furthermore, financial supports were both included and excluded in the calculations to show a wide range of alternatives.

\section{Key results and discussion}

The most striking result was that it was only the organic production that reached full cost coverage, i.e. revenues $\geq$ expenses. The differences in profitability between diet $\mathrm{M}$ and $\mathrm{H}$ were small in organic production. Diet $\mathrm{M}$ performed slightly better compared with diet $\mathrm{H}$ in all districts and herd sizes except in the NF district with the support, where it was more profitable to use diet $\mathrm{H}$. This result was probably due to higher price on concentrates in the northern part of the country. Cultivation of grains in the north is 
challenging due to the short vegetation period. In contrast, the conditions for cultivation of leys are favourable in the north. Maximum profitability in organic production with support was shown with 160 cows in the NF district, and without the support with 160 cows in the SP district. The results showed that overall there were large benefits in economies of scale with higher profitability using 160 cows over 80 cows in production. The benefits of larger herds are associated with less labour hours and lower building costs per cow/year and greater milk price because of large volumes of delivered milk. In conventional production, diet $M$ showed maximum profitability and diet $L$ minimum profitability in all districts and herd sizes both with and without support. The main results also reflect the high costs of housing the animals and the high costs of labour in Sweden, as well as the strong dependency of financial support by the farmers.

\section{How work was carried out?}

In the animal experiment, 92 cows were used, randomly assigned to one of three diets that differed in forage proportion. During the first 12 weeks of the lactation, the cows were fed grass silage ad libitum and the amount of concentrate was adjusted to meet the target proportion of forage of $40 \%$ in diet $L$ representing a conventional diet, and $50 \%$ in diet $\mathrm{M}$ and $\mathrm{H}$ representing two different organic diets. From lactation week 13 until drying off, the forage proportions were gradually increased as lactation proceeded to eventually reach $50 \%, 70 \%$ and $90 \%$ in diet $\mathrm{L}, \mathrm{M}$ and $\mathrm{H}$, respectively. The results from the animal experiment showed similar milk yields on diet $L$ and $M$, but significantly lower yield on diet $\mathrm{H}$. The milk composition was not significantly different among the diets, although the fat content increased with increasing forage proportion (from $4.3 \%$ to $4.5 \%$ ). Higher concentrations of unsaturated fatty acids, which are beneficial to human health and tend to give e.g. more spreadable butter, are often shown in high forage diets. An important prerequisite for the similar results in milk yield on diet $L$ and $M$ was the high nutritional quality of the grass silage when replacing concentrate with forage. The profitability of milk production was expressed as incomes minus expenses per cow and year on each diet and mainly based on regional enterprise budgets from the Swedish University of Agricultural Sciences (Agriwise 2012). Supports from the EU (environmental payment for cultivation of leys, support for less favoured areas and payment for organic production) were both included and excluded in the calculations.

\section{References}

Agriwise 2012. Regional Enterprice Budgets. Swedish University of Agricultural Sciences, Department of Economics, Uppsala, Sweden.

Patel M 2012. Effects of Increasing the Proportion of High-Quality Grass Silage in the Diet of Dairy Cows. Dissertation 2012:80. Swedish University of Agricultural Sciences, Uppsala, Sweden. 